\title{
Regulation 2030 First Steps of A Journey
}

Since the dawn of time, people tried to predict the future in order to gain some control over what may come. There is a vested interest in attempting to forecast events and situations that may have an effect on individuals and organizations-nursing regulators are no different. The question is, are we "masters of our fate" or victims of circumstances yet to come? William Shakespeare said, "It is not in the stars to hold our destiny but in ourselves."

During the NCSBN-hosted Regulation 2030 Conference last Fall, nurse leaders and regulators from around the world endeavored to help chart the future of nursing regulation. Participants came from 8 countries, 17 U.S. states, the District of Columbia, and 2 U.S. territories - as well as representatives from the American Association of Colleges of Nursing, U.S. Federal Trade Commission, Federation of State Boards of Physical Therapy, Federation of State Medical Boards, National League for Nursing, Organization for Associate Degree Nursing, American Nurses Association, National Federation of Licensed Practical Nurses, and Organization of Economic Cooperation Development (OECD)-undertook the task of trying to understand where nursing regulation is headed (National Council of State Boards of Nursing [NCSBN], 2016b).

In his opening remarks, NCSBN CEO David C. Benton challenged participants to do "out of the box" and audacious thinking about the challenges and opportunities of the future. He noted that nursing regulation is increasingly influenced by globalization, the growing complexity of the health care system, the emergence of new infectious diseases and the reemergence of older diseases such as tuberculosis, nurse mobility, and changing demographics and social behavior. This study presents the first steps of the journey toward forecasting and possibly controlling to some degree as much of the trends and issues that will lead us to a contemporary and effective approach to nursing regulation in 2030.

This study is presented in three steps. Step One, Bibliometric Review: Identifying Regulatory Themes and Emergent Trends, presents a bibliometric analysis of the peer-reviewed literature on professional self-regulation and occupational licensure, identifying and describing new and emergent trends, and issues facing nursing regulation and how they have evolved over time.

Step Two, Capturing the Essence: Development of Regulatory Concept Maps, interprets and formalizes into actionable items the 25 emergent trends identified in Step One. The experts from around the world who participated in the 2030 conference (listed above) interpreted these emergent trends for the future, along with their implications. They then projected what needs to be in place to deliver the emergent trends, identifying the impacts they will have on nursing regulation and the health care systems. Their interpretations, implications, and projections were then formalized by creating concept maps for each of the 25 emergent trends. These maps are presented in this Step Two.

Step Three, Analysis and Priorities: Developing a Modern, Effective Regulatory Framework, describes and reports on the analytical processes followed to synthesize the 25 concept maps in order to determine the key priorities for moving forward in developing an effective regulatory framework.

While vast quantities of data have been generated, analysed, and prioritized, further work is needed in terms of how the nursing profession and regulators, in particular, will respond to the findings both at the micro (individual trends) and macro (general theme) levels. As with most research, the work has generated questions and actionable steps that need to be addressed.

The data demonstrate the importance of collaboration, performance measures and metrics, governance, and data and technology. However, the totality of our knowledge about these subjects and whether there are sound evidence and best practices available to support them remains unknown. These become some of our most pressing topics for further research. As these subjects are explored, the concept maps should be revisited. A research program, based on these four areas-collaboration, performance measures and metrics, governance, and data and technology-is clearly necessary.

There is also a need for a major review of current regulatory policies and their associated processes. These should be examined for how they might support or disrupt the evolving directions of regulatory trends. For example, is there a need to revise the key pillars of the profession, the basic principles of the profession, and/or the model practice acts and related rules? The answers to these questions could be logical starting points for revision. 
Regulation is no different from any other aspect of today's society: It cannot remain dormant in light of the rapid pace of changing data systems, information security and use, and predictive analytics. All these technologies are important to regulators and offer additional areas for scientific exploration.

Historically, in response to high-profile regulatory failures, the government has initiated changes to the regulatory system. Today, regulators, must lead and bring about change through the development of an enhanced system based on regulatory performance metrics and measures. This will allow regulators to execute an evidence-based process that demonstrates accountability, efficiency, and effectiveness and establishes a model of professional regulation fit for today's complex, dynamic, digital era and beyond. 